\title{
Towards an updated view on the clinical management of pancreatic adenocarcinoma: Current and future perspectives (Review)
}

\author{
LEONEL PEKAREK $^{1-3}$, OSCAR FRAILE-MARTINEZ ${ }^{1,2}$, CIELO GARCIA-MONTERO $^{1,2}$, \\ MIGUEL A. ALVAREZ-MON ${ }^{1,2}$, JULIO ACERO ${ }^{2,4}$, LIDIA RUIZ-LLORENTE ${ }^{2,5}$, \\ NATALIO GARCÍA-HONDUVILLA ${ }^{1,2}$, AGUSTIN ALBILLOS ${ }^{1,2,6,7}$, JULIA BUJÁN ${ }^{1,2}$, \\ MELCHOR ALVAREZ-MON ${ }^{1,2,7,8}$, LUIS G. GUIJARRO ${ }^{2,5,8^{*}}$ and MIGUEL A. ORTEGA ${ }^{1,2,9^{*}}$
}

\begin{abstract}
${ }^{1}$ Department of Medicine and Medical Specialities, Faculty of Medicine and Health Sciences, University of Alcalá, Alcala de Henares, 28871 Madrid; ${ }^{2}$ Ramón y Cajal Institute of Sanitary Research, 28034 Madrid; ${ }^{3}$ Oncology Service, Guadalajara University Hospital, 19002 Guadalajara; ${ }^{4}$ Department of Surgery, Medical and Social Sciences, Faculty of Medicine and Health Sciences; ${ }^{5}$ Unit of Biochemistry and Molecular Biology, Department of System Biology, University of Alcalá, Alcala de Henares, 28871 Madrid; ${ }^{6}$ Department of Gastroenterology and Hepatology, Ramón y Cajal University Hospital, University of Alcalá, Ramón y Cajal Institute for Health Research;

${ }^{7}$ Biomedical Research Networking Center of Hepatic and Digestive Diseases, Institute of Health Carlos III, 28034 Madrid; ${ }^{8}$ Immune System Diseases-Rheumatology, Oncology Service and Internal Medicine;

${ }^{9}$ Cancer Registry and Pathology Department, Prince of Asturias University Hospital, Alcala de Henares, 28806 Madrid, Spain
\end{abstract}

Received July 14, 2021; Accepted September 3, 2021

DOI: $10.3892 / 01.2021 .13070$

\begin{abstract}
Pancreatic cancer has a dire prognosis and will represent the second leading cause of cancer death in the next 10 years. The multifactorial approach represents one of the main issues in controlling the extension of this neoplasm. In recent years, the characteristics of the tumor microenvironment, metastasis mechanisms and the relationship between immune system and neoplastic cells have been described, which has made it possible to understand the pathophysiology of pancreatic adenocarcinoma. Currently, there is a failure to provide an effective preventive method or early detection, so patients present with an advanced stage at the time of diagnosis. Despite numerous efforts, little progress has been made in clinical outcome and in improving survival in long term. Therefore, in the recent years, diverse diagnostic tests, treatments and possible approaches have been developed in the fields of radiotherapy, chemotherapy and surgery to find a combination of them that
\end{abstract}

Correspondence to: Professor Miguel A. Alvarez-Mon, Department of Medicine and Medical Specialities, Faculty of Medicine and Health Sciences, University of Alcalá, 19 Avenue de Madrid, Alcalá de Henares, 28871 Madrid, Spain

E-mail: maalvarezdemon@icloud.com

${ }^{*}$ Contributed equally

Key words: pancreatic cancer, serological markers, pancreatic cancer immunotherapies, pancreatic cancer diagnosis improves life expectancy in patients diagnosed with pancreatic cancer. At the moment, numerous clinical trials are being conducted to evaluate preventive diagnostic procedures such as serological markers or perfecting available imaging tests. On the other hand, implementation of immunotherapy is being studied in a neoplasm that has lagged in the application of this procedure since present possible treatments do not substantially improve quality of life. Therefore, the purpose of our study is to summarize the main progresses that have been made in the diagnosis, treatment and screening of this disease, explaining the limitations that have been observed and analyzing future prospects in the management of this illness.

\section{Contents}

1. Introduction

2. Current problem and pathophysiology

3. Diagnostic imaging: New approaches

4. Serological markers and CA19-9

5. Surgery is becoming less and less invasive

6 New chemotherapy treatment guidelines

7. Advances in radiotherapy treatment

8. Current strategies with immunotherapy

9. Conclusions

\section{Introduction}

In recent years, the incidence and mortality rates of pancreatic cancer have been growing along with the global population. 
Currently, adenocarcinoma of the pancreas represents $\sim 80 \%$ of pancreatic cancer cases and is one of the main causes of cancer-associated mortality (1). The increase in incidence and mortality, and the difficulty in the development of effective therapies led to pancreatic cancer being predicted as the second highest cause of cancer-associated death in developed countries by the year 2030. (2) One of the possible causes that explain a rise in the number of cases of pancreatic cancer in recent years could be related to an increase in the number of people in the general population with predisposing factors such as tobacco use, obesity or alcohol consumption; areas that a number studies have highlighted as risk factors in the appearance of premalignant lesions or chronic pancreatitis (3). The presence of diabetes, a disease on the rise in recent years, has been shown to be another important risk factor in the etiopathogenesis of pancreatic adenocarcinoma. In diabetes mellitus, a pro-inflammatory state is provoked where diverse factors derived from insulin and tumor growth act to promote neoplastic growth and alter numerous metabolic pathways in tumor cells (4). In reference to its incidence, there are differences between developed and developing countries, affecting the former to a greater extent, with a global incidence of $\sim 5$ patients per 100,000 inhabitants (5). Despite the existence of various types of approaches in treating pancreatic adenocarcinoma (including laparoscopic surgery, chemotherapy, radiotherapy and immunotherapy), the mortality rate has not decreased as it has in other types of malignant neoplasms (6). This is due to the difficulty of early diagnosis and effective treatment, which means that most patients are at an advanced disease stage at the time of diagnosis. Today, according to different studies, the 5-year survival rate when the patient is diagnosed ranges between 2 and 10\%, with a mean survival time of 6 months, given that most patients present with disseminated disease with metastases in multiple organs (7-9). Several risk factors have been described that increase the probability and worsen the prognosis in these patients. Among them are obesity, tobacco use, alcohol consumption and premalignant lesions, which act in a multifactorial manner, but to date, there are no screening programs in high-risk patients that have been able to demonstrate effectiveness in decreasing mortality or improving survival (10). The interaction between different agents in other digestive tract neoplasms allows to support an etiopathology that relates external agents with the appearance of invasive neoplasia (e.g., processed red meat in colon cancer and human papilloma virus in rectal cancer.). However, in pancreatic adenocarcinoma, the risk factors described are present in $\sim 40 \%$ of the patients and genetic causes are present in $10 \%$ of the patients, with a variety of mutations in the BRCA genes, Lynch syndrome genes or familial adenomatous polyposis genes (11). There are uncommon varieties of pancreatic exocrine tumors, such as pancreatoblastoma, where the risk factors described are mainly genetic, such as Beckwith-Wiedemann syndrome or familial adenomatous polyposis (12). Most of the studies that analyze the risk factors present in exocrine tumors, including ductal adenocarcinoma, which is the most studied due to its frequency, conclude that risk factors prevalent in the population such as older age, obesity or tobacco use are common in all different types of pancreatic neoplasias, and they worsen the prognosis. However, in pancreatic endocrine tumors (insulinoma, glucagonoma and VIPoma, among others), a stronger genetic predisposition and a history of gene mutations in multiple endocrine neoplasms genes, such as in von Hipple-Lindau syndrome or neurofibromatosis, are predominant risk factors (13). On the other hand, in recent years, there have been numerous advances in the approach to this disease. Multiple diagnostic, microsurgery and radiochemotherapy methods have been described and are being studied in order to be implemented in daily clinical practice, in addition to pursuing the development of new follow-up techniques for the early diagnosis of this disease (14). In recent years, the study of possible therapeutic targets has been promoted in order to be able to apply targeted therapy against this type of tumor. Despite the advances made, further efforts are required to improve the treatment and early diagnosis of pancreatic cancer, which affects numerous individuals fatally each year and has a poor prognosis at the time of diagnosis.

Therefore, the purpose of the present review is to describe the novelties, updates and future perspectives in the diagnosis, treatment and management of patients affected by pancreatic cancer.

\section{Current problem and pathophysiology}

In 2020, pancreatic cancer represented the 12 th most common cancer and the 7th leading cause of cancer-associated death in the world (15). Numerous modifiable and non-modifiable risk factors have been described. Among them, the most important and relevant modifiable risk factors are age, since $90 \%$ of patients are $>50$ years old, alcohol consumption, obesity, being male, diabetes and tobacco use (16). The most frequent histological variation is ductal adenocarcinoma of the pancreas, located primarily in the head of the pancreas (17). In addition to the ease of the tumor itself with regard to metastatic spread and vascular invasion of the tumor, the location in the head of the pancreas makes the surgical approach even more difficult, since it causes the surgical intervention to be highly aggressive, resulting in excessive mortality and morbidity rates $(18,19)$. It should be noted that there are several types of premalignant lesions (such as pancreatic intraepithelial neoplasia, papillary intraepithelial mucinous neoplasia and mucinous cystic neoplasia) that act as an intermediate stage from the appearance of mutations in the pancreatic mucosa to the appearance of a true invasive neoplasm (20). In numerous cases, the choice of surgical intervention is difficult, as most premalignant lesions, $\sim 90 \%$, do not lead to a true pancreatic malignancy, which is why patients could be subjected to aggressive techniques that are not exempt of risk (21). On the other hand, it cannot be overlooked that although attempts have been made to propose population screening measures, effective screening methods have not been implemented due to the low incidence of the disease (22). This low incidence is due to the lack of detection markers that are directly related to invasive pancreatic neoplasms, the fact that imaging tests do not detect tumors of diminutive sizes and due to the large susceptible population with multiple risk factors, making it difficult to screen for this disease in the initial stages. Due to the complexity in diagnosis, the lack of initial symptoms and the aggressiveness of this type of tumor itself, the vast majority of patients are diagnosed in advanced, unresectable and non-curative stages, undergoing 
considerably aggressive chemotherapy regimens that do not substantially improve the life expectancy, and with a poor prognosis from the time of diagnosis. In order to understand the complexity of the treatment and the aggressiveness of pancreatic adenocarcinoma, the underlying pathophysiology of the tumor itself, which has been completely described, cannot be ignored. In recent years, important advances have been made in the discovery of diverse mutations, markers and pathophysiological bases, which has allowed us to relate histological, molecular and genetic alterations with the clinical manifestations and the outcome of this disease (23). It must be noted that numerous pathological types of pancreatic cancer have been described (including insulinomas, acinar cell carcinoma and hepatoid carcinoma); however, the present review focuses on the oncogenesis of the most common type, ductal adenocarcinoma. Ductal adenocarcinoma represents $>80 \%$ of pancreatic cancer cases and affects the mucous cells of the pancreatic ducts (24). In the first instance, the multiple exogenous toxic agents described, such as tobacco, alcohol or other substances, cause mutations in the Kirsten rat sarcoma virus (KRAS) gene and modify the expression of proteins related to the RAS/MAPK pathway (25). The KRAS mutation generates a series of modifications in cell repair mechanisms and in growth regulation due to the interaction with different growth factors (mainly transforming growth factor $\beta$ and fibroblast growth factor) and the signaling pathways, mostly MAPK and PI3K/AKT, that promote the cell proliferation of the tumor line $(26,27)$. This lesion begins as a tissue dysplasia known as pancreatic intraepithelial neoplasia. It is recognized that the dysplastic lesion acquires different mutations (in p16, p53, DPC4 and BRCA2, among others) and alterations in differentiation, which eventually will turn it into an invasive neoplastic lesion (28). Furthermore, tumor cells interact with pancreatic stellate cells, which are myofibroblastic-like cells with autocrine and paracrine functions that cause alterations in the extracellular matrix, generating pancreatic fibrosis or desmoplasia (29,30). All this microenvironment generated around the neoplastic cells causes local immunosuppression, as well as angioproliferation that favors vascular invasion and therefore promotes extrapancreatic metastasis (31). At present, this interaction between tumor cells and extracellular tissue is still being described, and it has been observed that numerous signaling pathways that determine tumor aggressiveness are involved in their complex relationship (32). Tumor cells generate chemoresistance against the main available therapeutic agent, gemcitabine, which inhibits the effectiveness of one the main treatment approaches for this cancer. These chemoresistance mechanisms are produced by the tumor microenvironment, by desmoplasia or by the invasive cells itself, and are based on alterations in nucleoside transporters such as decreases in human equilibrative nucleoside transporter 1 , deoxycytidine kinase or ribonucleotide reductase expression, therefore decreasing entry of gemcitabine into the tumor cells $(33,34)$. Other alterations at the level of cellular expression, such as epithelial-mesenchymal transition (EMT), generate changes in the interaction of the tumor with the surrounding cells, favoring its spread and hindering the action of the diverse chemotherapeutic lines (35). The EMT alters from cell metabolism to gene expression by producing different transforming factors like SNAIL, PRRX or Twist and different types of microRNA, which are implicated in modifications of various metabolic pathways like glycolysis, promoting the Warburg effect or the tricarboxylic acid cycle causing the tumor to act as a mesenchymal invasive and chemoresistance tissue, so that even though the different antineoplastic drugs are acting, the cell can continue to proliferate and invade adjacent tissues $(36,37)$.

Overall, although the treatment of different malignant neoplasms has progressed in recent years through increasingly more precise diagnostic techniques, and more targeted and less toxic treatments, the complexity of the pathophysiology of pancreatic cancer forces the scientific community to continue investigating new lines in prevention, early diagnosis and therapeutic targets in order to improve the prognosis in patients affected with ductal adenocarcinoma of the pancreas.

\section{Diagnostic imaging: New approaches}

One of the primary diagnostic problems of pancreatic cancer lies in the difficulty of an early diagnosis, and in cases in which advanced disease is suspected, most of the time, imaging tests such as computed tomography, magnetic resonance imaging, cholangiopancreatography and intraductal ultrasound are used without the need for histological confirmation (38). Another problem in its follow-up is based on the difficulty of detecting metastases in different organs, and the current techniques usually do not offer substantial results when it comes to detecting tiny lesions in order to allow an early treatment approach (39). It should also be mentioned that in cases in which premalignant lesions are suspected, the confirmation of the neoplasia must be histological. Invasive tests using a biopsy are not without risks and can have fatal consequences in cases in which abdominal bleeding or pancreatitis is caused in patients already compromised by their pancreatic neoplasia and comorbidity $(40,41)$. All this means that new techniques have been developed for the diagnosis of early stages. For example, in screening, a low-cost, reproducible and fast technique with few possible complications, such as transabdominal ultrasound, would be useful. Although this technique is not very effective in detecting lesions $<2 \mathrm{~cm}$ in diameter, the radiological ultrasound reading of vascularization patterns of pancreatic malignant lesions and the utility of ultrasound with the application of contrast is being protocolized (42). In reference to tomography, high-resolution multidetector tomography scans have been used combined with different algorithms in image reconstruction to improve the visualization of the pancreatic parenchyma and its vascular perfusion, and therefore to identify neoplastic lesions $1-2 \mathrm{~cm}$ in diameter (43). It is worth bearing in mind that at the stromal level, the desmoplastic and fibrotic changes surrounding the pancreatic lesion have vascular perfusion characteristics that could be visible on multislice tomography scans (44). It should also be noted that when using magnetic resonance, new approaches have been made, for example, using an anti-mesothelin antibody. Mesothelin is an antigen overexpressed in pancreatic tumor cells and it has been used as a possible therapeutic agent combined with nanoimmunoliposome doxorubicin-delivery particles to link iron oxide contrasts and chemotherapy for magnetic resonance imaging and target therapy. This allows 
better visualization of the pancreatic lesion in this type of imaging tests and could provide a new therapeutic perspective; however, their clinical utility is still under study $(45,46)$. Similarly, novel advances have been described in the use of tracers in positron emission tomography to highlight tumors and metastatic tissues in these patients, and enable their detection even in early stages, improving the staging of the tumor, assessing recurrence, establishing the differential diagnosis with inflammatory lesions and planning the biopsy of the tissue that may be susceptible to histological study (47). The approach that has shown the greatest sensitivity and specificity to localize lesions $<1 \mathrm{~cm}$ continues to be endoscopic ultrasound (48). Although it is explorer-dependent, it is greatly beneficial in cases of differential diagnosis between pancreatic cystic neoplasms and malignant neoplasms, preventing pancreatitis, hemorrhage, perforation or fever by using thinner needles for aspiration purposes. The technique also permits the acquisition of material such as DNA, mRNA or microRNA, which can provide the analysis of diverse immunomarkers such as KRAS, protein S-100 and insulin receptor substrate 4, which plays an important role in the promotion of tumor growth in different malignancies. This is an advantage for the molecular diagnosis of pancreatic neoplasia in an obtained sample (49-51). Another invasive test that is most used in diagnosis is endoscopic cholangiopancreatography. Even though it is a test with high sensitivity and specificity, the greatest disadvantage of this technique is the risk of causing pancreatitis (2-9\% of patients), cholangitis or hemorrhage that can compromise the patient (52). Smaller endoscopic ultrasound devices are therefore being developed to decrease the risk of complications during the procedure (53). It must also be highlighted that even more sensitive and less invasive diagnostic tests, such as intraductal ultrasound, have been developed in recent years (54). This technique is performed by endoscopy with markedly small probes and enables the intrapancreatic ducts to be detected and most of the intraluminal structures of the pancreas to be visualized to discover all possible pancreatic neoplastic lesions (55). This test is especially valuable when planning surgical intervention, since wide tumor invasion of the ducts, which could not have been visualized with conventional radiology techniques, would contraindicate a curative resection (56). It should not be forgotten that pancreatic cancer is a metastatic disease that requires multiple diagnostic tests to clarify locoregional invasion and metastatic tumor staging. As a consequence, it would be especially effective to integrate different types of tests that were least invasive with a high sensitivity and specificity in the diagnosis of pancreatic cancer.

\section{Serological markers and CA19-9}

In recent years, numerous oncological markers, which are usually glycoproteins released by the tumor or produced after the interaction between the immune system and neoplastic cells, have been described. These glycoproteins can be measured in the peripheral fluids and assist the diagnosis of a multitude of malignant neoplasms. Among the different tumor markers, such as prostate-specific antigen in prostate cancer, carbohydrate antigen CA15.3 in breast cancer and CA125 in ovarian cancer, it is worth highlighting the use of
CA19-9 in pancreatic cancer (57-59). The increase in CA19-9 by values $>300 \mathrm{U} / 1$ drives its positive predictive value to $90 \%$ (that is, if a patient tests positive there is a $90 \%$ chance that they in fact have pancreatic cancer) (60). The sensitivity of $80 \%$ and the specificity of $\sim 90 \%$ is one of the main problems of this marker; although a priori it seems to be useful, it is also expressed in other types of malignant processes such as colon, esophageal and liver cancer, or non-malignant disease processes such as chronic pancreatitis, liver cirrhosis and cholangitis (61). Furthermore, a percentage of the general population (5-10\%) does not possess the Lewis antigen and as a result, CA19-9 is not expressed in these patients when pancreatic cancer is present (62). All this means that only $\leq 65 \%$ of patients with pancreatic ductal adenocarcinoma susceptible to curative resection have elevated values of this marker (63). Therefore, the low sensitivity of CA19-9 to detect pancreatic malignant neoplasms is relegated to the follow-up of recurrence, to analyze the response to chemotherapy or to be used as a diagnostic orientation in conjunction with imaging tests (64). That is why in recent years an attempt has been made to identify new biomarkers for early diagnosis that can also be used in screening programs in patients with various risk factors. For example, different tumor growth markers have been described for use in pancreatic juice (carcinoembryonic antigen, Duke pancreatic monoclonal antigen type 2, cathepsin E and KRAS), saliva (KRAS, methyl-CpG binding domain protein 3 like 2 and dolichyl-phosphate mannosyltransferase subunit 1, catalytic), fecal matter (KRAS mutated, CA19-9 or CA242) or in combinations between them to improve the sensitivity and specificity, but none of them have been put into clinical practice or evaluated in large population studies to assess their positive predictive value (65-68). There are a number of reasons for this. On the one hand, the incidence of pancreatic cancer is not high enough to demonstrate effectiveness in daily clinical practice. On the other hand, taking such samples as pancreatic juice is not without risks since the sample is taken by cholangiopancreatography. It must be added that all the genetic, metabolic and tissue changes produced in tumor cells cause patients to present a great variety of molecular alterations, mutations and aberrations in the expression of different antigens. These variations make it even more difficult to validate a single marker or battery of markers in the diagnosis of pancreatic cancer (69). The ideal scenario would be the combination of other markers that allows us to detect initial stages. Numerous studies have assessed the simultaneous measurement of diverse markers, combining CA19-9 and others to detect those $5-10 \%$ patients with negative CA19-9 or improve the positive predictive value. A number of these studies have been used to compare individuals with pancreatic cancer versus healthy controls, early versus advanced stages of cancer, or invasive versus non-invasive disease, obtaining promising receiver operating characteristic (ROC) curves (70,71). It should be considered that the ROC curve measures the validity of a diagnostic test by assessing its sensitivity and specificity, with 1 being the maximum value $(72,73)$. The results of these studies are encouraging in the early diagnosis of the disease. For example, the combination of cell migration-inducing and hyaluronan-binding protein with CA19-9 made it possible to obtain values of 0.94 in the ROC curves (74). Another example is the use of thrombospondin 1 
and 2 together with CA19-9 in the blood, where ROC curves of 0.96 and 0.86 were obtained, respectively $(75,76)$. In addition, markers can not only be measured in blood, but in other samples too; for example, the use of markers such as lymphatic vessel endothelial hyaluronan receptor 1, islet of Langerhans regenerating protein and trefoil factor 1 in urine demonstrated ROC curve values of 0.93 for the diagnosis of early stages of pancreatic adenocarcinoma (77). At present, these markers are still under study in order for them to be used in the high-risk population or in those patients with dysplastic lesions of the pancreas. Also, their cost/benefit balance is being evaluated to determine if they would benefit the numerous patients who are in the initial disease stages and may be candidates for curative resection.

\section{Surgery is becoming less and less invasive}

Despite all the advances that have been made in the chemoradiotherapy treatment of pancreatic cancer, the only curative treatment that exists in this type of tumor is surgical resection. Since the neoplasm is diagnosed in late stages, only $10-20 \%$ of patients are candidates for curative resection $(78,79)$. It should be noted that although the size of the tumor is not important, the presence of metastasis or the invasion of peripancreatic vessels (including the common hepatic artery, mesenteric vein and portal vein, among others) significantly limits the possibility of intervention (80). Currently, the intervention being performed is a cephalic duodenopancreatectomy, which in the past was characterized as an aggressive surgical approach that produced high mortality with little expectation of improvement in survival prognosis (81). For a long time, this technique was unused due to its high mortality rate (up to $30 \%$ ), but it is currently performed by expert surgeons with a postoperative mortality of $3-5 \%$. However, in the postoperative period, the infection rate of the surgical wound, anastomotic dehiscence and various complications are common (82). The technique is not without risks, which is why new minimally invasive techniques have been developed. For years, this has included the use of laparoscopy in distal pancreatectomy in lesions in the tail of the pancreas, which has reduced intraoperative bleeding, although it has not been able to improve the long-term prognosis (83). On the other hand, the use of robotics with minimally invasive interventions has been studied and its results have been compared with laparoscopy, with very different outcomes according to the different studies. $(84,85)$ In some of them, a greater number of complications (fistula, hemorrhage and perforation) were observed with the robotic intervention; however, it must be remembered that there are few specialists trained in this type of technique and that this type of approach requires a high level of training that may not be available at a number of centers $(86,87)$. Likewise, it cannot be overlooked that the greatest limitation when considering surgical intervention is vascular invasion. Thus, the vascular approach is as important as the surgical resection itself. Usually, pancreatic lesions diagnosed by imaging tests with vascular invasion greater than circumferential $180^{\circ}$ of the superior mesenteric vein, portal vein, common hepatic vein or its branches contraindicate surgery $(88,89)$. Thanks to advances in vascular radiology for detecting tumor invasion of the different vascular territories, combined arterial or venous reconstruction therapies are being developed in the segments affected by the tumor. In recent years, some of the main surgical improvements that have been developed to increase the number of patients susceptible to a curative option are the numerous surgical techniques for approaching venous invasion of the pancreatic adenocarcinoma. For example, venorrhaphy, patch closure, interposition conduit and primary anastomosis have been used in the vascular approach of venous invasion (90). These techniques have made it possible to change the prognosis of patients with pancreatic adenocarcinoma, allowing the patient to become a candidate for a surgical option (91). It is true that there are currently no biological therapies, such as angiogenesis inhibitors, that are used in pancreatic adenocarcinoma, so it would be notable to study this therapeutic option in this type of neoplasia. Even so, in recent years, the therapies that act on the vascular invasion of adenocarcinoma have multiplied and numerous approaches with complex vascular techniques are being developed. Different studies have shown that both venous and arterial reconstruction improve survival compared with palliative surgeries or chemoradiotherapy $(92,93)$. In recent years, palliative techniques have been developed to limit the duodenal-biliary obstruction that causes locoregional tumor invasion. Possible approaches, including external biliary drainage, surgical biliary diversions or metal stents, are useful in cases where survival time is limited to 3-6 months, and these approaches permit obstructive symptoms such as jaundice or pancreatic insufficiency to be decreased in the final stages of disease for these patients (94-96). It should also be noted that the invasion of the nerve plexuses generates neuropathic pain of great intensity that may be resistant to drug treatment with analgesics (97). A celiac plexus neurolysis can therefore be performed by endoscopic ultrasound, which can be considered an optimal analgesic treatment (98). Therefore, even though surgery plays a very relevant role in the cure of pancreatic cancer, few patients can benefit from this type of intervention, which is also not exempt of risks and complications.

\section{New chemotherapy treatment guidelines}

Classically, chemotherapy treatment has been the basis of the approach in metastatic disease of pancreatic cancer since most patients $(90 \%)$ are not considered suitable for curative surgical intervention. The present reference scheme for chemotherapy treatment has been the use of gemcitabine (99). Although the use of gemcitabine has been tested in combination with different chemotherapeutics, such as 5-fluorouracil, capecitabine and taxane, these have failed to demonstrate an increase in median survival rate $(100,101)$. That is why new combinations of different regimens were investigated, included current use of first-line FOLFIRINOX and Abraxane (102). FOLFIRINOX is based on the union of folinic acid, 5-fluorouracil, irinotecan and oxaliplatin, all of them with broad antineoplastic activity in pancreatic cancer and whose combination has been shown to improve survival compared with the use of gemcitabine alone in patients with a good baseline state, with scores of 0-1 on the Eastern Cooperative Oncology Group (ECOG) 
scale $(103,104)$. One of the problems generated by the results of the clinical trials with FOLFIRINOX was an increase in toxicity compared with use of gemcitabine alone. For example, severe neutropenia, thrombocytopenia, diarrhea, vomiting and peripheral neuropathy were reported in up to $51.6 \%$ of the patients treated with FOLFIRINOX (105). It should be noted that this scheme has been studied in patients with few comorbidities, who also had a good baseline state (with an ECOG score of 0-1). In daily clinical practice, the large majority of patients do not present with a baseline state good enough to be treated with aggressive regimens (106). Therefore, FOLFIRINOX competes with the combination of nab-paclitaxel plus gemcitabine as as the front-line treatment, thereby two possible options to treat metastatic disease (107). Nab-paclitaxel is a molecule composed of the union of albumin with paclitaxel where albumin participates in endothelium caveolae transcytosis of plasma components. In vitro, it has been shown that the presence of albumin promotes the transport of paclitaxel through pancreatic tumor cells, allowing paclitaxel to act on the tumor cells (108-110). It should be noted that one of the difficulties in the treatment of pancreatic cancer is the difficulty of the chemotherapeutic agent to act at the level of the tumor parenchyma. The combination of nab-paclitaxel with gemcitabine in patients with metastatic disease has been shown to improve survival compared with the use of gemcitabine alone, and it also confers fewer adverse effects compared with FOLFIRINOX (111). In addition, this therapy is useful in patients with comorbidities or with an ECOG score greater than 1 who are not candidates for the use of FOLFIRINOX $(112,113)$. Currently, gemcitabine as a single treatment could be used as an non-aggressive treatment in those patients who have metastatic disease with poor general condition in which other chemotherapy regimens have not shown progress $(E C O G \geq 2)(114)$. Despite the fact that a number of improvements have been made in terms of the toxicity of the different chemotherapeutic lines, currently, first-line combinations are not able to generate mean survival rates of more than 12 months (115). In recent years, there have been numerous clinical trials where the use of neoadjuvant chemotherapy in the treatment of pancreatic cancer has been analyzed. With neoadjuvant therapy, doses of FOLFIRINOX or gemcitabine are given to decrease tumor size and reduce the probability of presenting with undetected micrometastases caused by pancreatic neoplasia, and thus reinforce the elimination of all neoplastic dissemination after a surgical intervention (116). In this sense, neoadjuvant therapy was evaluated in a meta-analysis of 38 studies performed for this purpose and it was found that the use of neoadjuvant chemotherapy enhanced overall survival. However, the rates of surgical resection were lower than in those patients treated directly with surgery or patients with borderline tumors (117). One of the possible causes is that patients who are candidates for neoadjuvant treatment have lesions that affect the edges of resection or patients could have tumors that are difficult to approach due to invasion or previous comorbidities of the patient themselves. Therefore, the patients are initially candidates for neoadjuvant treatment with subsequent surgery, but during the application of chemotherapy regimens and prior to the intervention, tumor progression is presented (118). Despite receiving neoadjuvant therapy, patients are later rejected for surgical intervention, which would explain why patients who are candidates for neoadjuvant treatment do not end up being operated on and why treatment is based only on chemotherapy. Another possible cause is that nowadays neoadjuvant chemotherapy regimens are not well defined, and the clinical studies performed have not found an optimal chemotherapy association to be used as neoadjuvant therapy (119).

\section{Advances in radiotherapy treatment}

Another possible approach to pancreatic cancer includes the use of radiation therapy. The use of radiation therapy to treat pancreatic cancer has long been relegated to the background. This is due to the inability to give effective doses in the pancreatic territory caused by the resistance of the tissue itself, the intraperitoneal location and the presence of underlying organs, which generates a very wide target volume for the use of radiotherapy. Radiotherapy also has important adverse side effects, including intraperitoneal fibrosis and damage to vital organs $(120,121)$. In recent years, therapies have been developed that allow the target volume to be reduced thanks to the use of stereotactic radiotherapy. External stereotactic radiotherapy makes it possible to limit the amount of irradiated tissue in a very precise way, while administering very high doses of radiotherapy without increasing the damage to peripheral organs (122). Although current evidence is limited, various authors from the American Radiology Oncology Association have proposed an updated guideline on the use of radiation therapy in pancreatic cancer (123). For example, benefits have been found when using stereotactic radiotherapy as adjunctive treatment in high-risk patients with affected margins after surgical resection or with extensive lymphatic invasion (124). Likewise, the usefulness of radiotherapy as a neoadjuvant treatment has been demonstrated together with the use of chemotherapy in patients with borderline tumors that affect the resection border (125). Despite being a technique that has reduced the risk of injury to adjacent organs or the presence of pseudoaneurysms, this procedure has been associated with numerous gastrointestinal adverse effects such as nausea, vomiting and diarrhea, although they are usually temporary and are directly related to the radiation therapy sessions (126). It must be remembered that this approach currently has numerous clinical trials underway to assess both radiotherapy doses and assess improved long-term survival benefits. For this reason, it is necessary to continue investigating the new options that radiotherapy can provide in order to reduce recurrence of the disease and control the metastatic progression.

\section{Current strategies with immunotherapy}

In recent years, immunotherapy has taken on a relevant role in the treatment of various solid tumors and has made it possible to improve survival in these patients by creating targeted therapies that act on a wide variety of therapeutic targets. In some cases, immune cell activity against programmed death-ligand 1 (PDL1) is promoted, such as in non-small cell lung cancer, cell division is blocked, such as BRAF in melanoma, or cell proliferation is limited, such as when using the tyrosine kinase 
inhibitor imatinib in chronic myeloid leukemia (127). For this strategy to be effective, the immune system must recognize tumor neoantigens, which differ from normal cells, to initiate an immune response and be able to act in a directed manner against the tumor without affecting healthy cells (128). The larger the variability of these neoantigens the greater the immunogenicity and the higher the immune response. In the specific case of pancreatic cancer, one of the main problems encountered in recent years is the low variability of tumor antigens compared with that in other tumors (129). While some tumors such as colon and lung tumors present with 100-1,500 neoantigens, pancreatic cancer presents with 10-60 $(130,131)$. Furthermore, the histological changes in mesenchymal tissue and the desmoplastic reaction further limit the immune system-tumor cell interaction since the immunosuppression generated by the presence of local regulatory lymphocytes is favored (132). This has led to the fact that single treatment with immunotherapies such as anti-CTLA4 or -PDL1 have not shown benefits according to different clinical trials comparing the treatments with classical gemcitabine regimens $(133,134)$. On the other hand, the association of immunotherapy with chemoradiotherapy has been studied, finding partial responses with the use of gemcitabine and ipilimumab (135). Different studies have proposed that the combination of immunotherapy with chemoradiotherapy promotes immunogenicity due to a decrease in immunoregulatory lymphocytes. It has been shown in pancreatic cancer that regulatory lymphocytes are present and are responsible for localized immunosuppression, and therefore limit the immune reaction (136-138). Moreover, another of the strategies proposed in recent years is the use of vaccines, where tumor antigens from neoplastic cells are used. For example, one of the best-studied vaccines, the GVAX vaccine against allogeneic antigens from neoplastic pancreatic cells irradiated to promote an immune interaction, has been shown in combination with immunochemiradiotherapy to increase survival time in preliminary results of different clinical trials, such as NCT03161379 or NCT02648282, that are still ongoing (139).

Numerous agents such as viruses, bacteria and fungi are also being used to continue exploring new mechanisms that facilitate the exposure of tumor antigens to the immune system through the use of vaccines (140). In the same way, immune cell therapies are being used such as chimeric antigen receptor therapy in which $\mathrm{T}$ lymphocytes are genetically modified to express the chimeric antigen receptor that recognizes a specific tumor antigen (141). This procedure involves the extraction of $\mathrm{T}$ lymphocytes from the patient and the subsequent reinfusion of modified cells, although it is still under study since it is not a risk-free technique, as it causes immune hyperstimulation that can be fatal in some cases (142).

Various therapeutic agents are being developed whose targets are different points of the disease. For example, studies have been conducted the efficacy of olaparib, which affects the repair of the tumor cell DNA by inhibiting the enzyme poly ADP ribose polymerase, pamrevlumab, which plays a role by inhibiting the proliferation of the extracellular matrix, and tyrosine kinase inhibitors such as devimistat that acts different mitochondrial metabolic pathways of the tumor cells (143-145). All these therapies are currently under study, although preliminary results show that the combination of most of these approaches together with polychemoradiotherapy improves survival and tumor resectability, and decreases the recurrence of pancreatic adenocarcinoma.

\section{Conclusions}

Numerous limitations have been discovered over time with regard to the treatment, diagnosis and prevention of different tumors. Stereotactic radiotherapy, immunotherapy, cell therapy or inhibitors of metabolic pathways are all now used in daily clinical practice, and these innovative approaches represent one of the main advances in the multidisciplinary management of cancer patients. Even though a wide variety of therapeutic agents have been developed, which have substantially improved the quality of life and prognosis of most patients with tumors, pancreatic cancer research continues to be one of the leading frontiers in terms of improving patient life expectancy with new therapies. The main limitation in performing clinical trials in pancreatic cancer is based on the low incidence and high mortality rates of this disease. Significant conclusions cannot be reached in a population where great variability is found in different disease factors, such as numerous tumor markers or multiple therapeutic approaches, and where every individual has to be carefully studied. However, in recent years, the numerous biomedical research advances on pathophysiology, and molecular and therapeutic targets represent a glimmer of hope in these patients whose disease continues to have a poor prognosis.

\section{Acknowledgements}

Not applicable.

\section{Funding}

The study was supported by the Comunidad de Madrid (B2017/BMD-3804 MITIC-CM) and HALEKULANI, S.L.

\section{Availability of data and materials}

Not applicable.

\section{Author's contributions}

MAO, LP, OFM, CGM, JA, MAAM, LRL, NGH, AA, JB, LGG and MAM were involved in the writing of the original draft and in the literature search. MAO, LP, OFM, AA, NGH, MAM, JB and LGG were involved in the writing, reviewing and editing of the manuscript. AA, LGG, MAM and LGG provided supervision. MAM, AA, JB, JA, LGG and MAAM provided resources. MAO, LG, OFM, CGM, LRL, LGG, MAM and MAAM were involved in the conceptualization of the study. All authors read and approved the final manuscript. Data authentication is not applicable.

\section{Ethics approval and consent to participate}

Not applicable.

\section{Patient consent for publication}

Not applicable. 


\section{Competing interests}

The authors declare that they have no competing interests.

\section{References}

1. Ilic M and Ilic I: Epidemiology of pancreatic cancer. World J Gastroenterol 28: 9694-9705, 2016.

2. Rahib L, Smith BD, Aizenberg R, Rosenzweig AB, Fleshman JM and Matrisian LM: Projecting cancer incidence and deaths to 2030: The unexpected burden of thyroid, liver, and pancreas cancers in the United States. Cancer Res 74: 2913-2921, 2014.

3. Guren MG: The global challenge of colorectal cancer. Lancet Gastroenterol Hepatol 4: 894-895, 2019.

4. Li D: Diabetes and pancreatic cancer. Mol Carcinog 51: 64-74, 2011.

5. Yadav D and Lowenfels AB: The epidemiology of pancreatitis and pancreatic cancer. Gastroenterology 144: 1252-1261, 2013.

6. Ansari D, Tingstedt B, Andersson B, Holmquist F, Sturesson C Williamsson C, Sasor A, Borg D, Bauden M and Andersson R: Pancreatic cancer: Yesterday, today and tomorrow. Future Oncol 12: 1929-1246, 2016.

7. Maisonneuve P: Epidemiology and burden of pancreatic cancer. Presse Med 48: e113-e123, 2019.

8. Mizrahi JD, Surana R, Valle JW and Shroff RT: Pancreatic cancer. Lancet 395: 2008-2020, 2020.

9. McGuigan A, Kelly P, Turkington RC, Jones C, Coleman HG and McCain RS: Pancreatic cancer: A review of clinical diagnosis, epidemiology, treatment and outcomes. World J Gastroenterol 24: 4846-4861, 2018.

10. Poruk KE, Firpo MA and Mulvihill SJ: Screening for pancreatic cancer. Ann Surg 257: 17-26, 2013.

11. Capasso M, Franceschi M, Rodriguez-Castro KI, Crafa P, Cambiè G, Miraglia C, Barchi A, Nouvenne A, Leandro G, Meschi T, et al: Epidemiology and risk factors of pancreatic cancer. Acta Biomed 89: 141-146, 2018.

12. Vilaverde F, Reis A, Rodrigues $\mathrm{P}$, Carvalho A and Scigliano $\mathrm{H}$ Adult pancreatoblastoma-case report and review of literature. J Radiol Case Rep 31: 28-38, 2016.

13. Geurts JL: Inherited syndromes involving pancreatic neuroendocrine tumors. J Gastrointest Oncol 11: 559-566, 2020.

14. Zhang Q, Chen S, Zeng L, Chen Y, Lian G, Qian C, Li J, Xie R and Huang KH: New developments in the early diagnosis of pancreatic cancer. Expert Rev Gastroenterol Hepatol 11: 149-156, 2017.

15. Sung H, Ferlay J, Siegel RL, Laversanne M, Soerjomataram I, Jemal A and Bray F: Global cancer statistics 2020: GLOBOCAN estimates of incidence and mortality worldwide for 36 cancers in 185 countries. CA Cancer J Clin 71: 209-249, 2021.

16. Kolodecik T, Shugrue C, Ashat M and Thrower EC: Risk factors for pancreatic cancer: Underlying mechanisms and potential targets. Front Physiol 16: 415, 2014.

17. Haeberle L and Esposito I: Pathology of pancreatic cancer. Transl Gastroenterol Hepatol 27: 50, 2019.

18. Pereira NP and Corrêa JR: Pancreatic cancer: Treatment approaches and trends. J Cancer Metastasis Treat 4: 30, 2018.

19. Berardi R, Mandolesi A, Pellei C, Maccaroni E, Onofri A, Lucarelli A, Biagetti S, Alfonsi S, Caramanti M, Savini A, et al: Prognostic factors in pancreatic cancer: The role of perineural, vascular and lymphatic invasion and of Ca19-9. J Gastroint Dig Syst 3: 134, 2013.

20. Delpu Y, Hanoun N, Lulka H, Sicard F, Selves J, Buscail L, Torrisani J and Cordelier P: Genetic and epigenetic alterations in pancreatic carcinogenesis. Curr Genomics 12: 15-24, 2011.

21. Andrén-Sandberg A: Non-pancreatic cancer tumors in the pancreatic region. N Am J Med Sci 3: 55-62, 2011

22. Kaur S, Baine MJ, Jain M, Sasson AR and Batra SK: Early diagnosis of pancreatic cancer: Challenges and new developments. Biomark Med 6: 597-612, 2012.

23. Khan MA, Azim S, Zubair H, Bhardwaj A, Patel GK, Khushman M, Singh S and Singh AP: Molecular drivers of pancreatic cancer pathogenesis: Looking inward to move forward. Int J Mol Sci 6: 779, 2017.

24. Hruban RH and Fukushima N: Pancreatic adenocarcinoma: Update on the surgical pathology of carcinomas of ductal origin and PanINs. Mod Pathol 20 (Suppl 1): S61-S70, 2007.

25. Fang Y, Yao Q, Chen Z, Xiang J, William FE, Gibbs RA and Chen C: Genetic and molecular alterations in pancreatic cancer: Implications for personalized medicine. Med Sci Monit 31: 916-926, 2013.
26. Visani M, Acquaviva G, Leo AD, Sanza V, Merlo L, Maloberti T, Brandes AA, Franceschi E, Battista MD, Masetti M, et al: Molecular alterations in pancreatic tumors. World J Gastroenterol 7: 2710-2726, 2021.

27. Vernucci E, Abrego J, Gunda V, Shukla SK, Dasgupta A, Rai V, Chaika N, Buettner K, Illies A, Yu F, et al: Metabolic alterations in pancreatic cancer progression. Cancers (Basel) 18: 2, 2019.

28. Gil YR, Sánchez PJ, Velasco RM, García AG and Lobo VJ: Molecular alterations in pancreatic cancer: Transfer to the clinic. Int J Mol Sci 19: 2077, 2021.

29. Pandol S, Edderkaoui M, Gukovsky I, Lugea A and Gukovskaya A: Desmoplasia of pancreatic ductal adenocarcinoma. Clin Gastroenterol Hepatol 7 (Suppl 11): S44-S47, 2009.

30. Merika EE, Syrigos KN and Saif MW: Desmoplasia in pancreatic cancer. Can we fight it? Gastroenterol Res Pract 2012: 781765, 2012.

31. Annese T, Tamma R, Ruggieri S and Ribatti D: Angiogenesis in pancreatic cancer: Pre-clinical and clinical studies. Cancers (Basel) 18: 381, 2019.

32. Li S, Xu HX, Wu CT, Wang WQ, Jin W, Gao HL, Li H, Zhang SR, $\mathrm{Xu}$ JZ, Qi ZH, et al: Angiogenesis in pancreatic cancer: Current research status and clinical implications. Angiogenesis 22: 15-36, 2019.

33. Zeng S, Pöttler M, Lan B, Grützmann R, Pilarsky C and Yang H: Chemoresistance in pancreatic cancer. Int J Mol Sci 11: 4504, 2019.

34. Grasso C, Jansen G and Giovannetti E: Drug resistance in pancreatic cancer: Impact of altered energy metabolism. Crit Rev Oncol Hematol 114: 139-152, 2017.

35. Wang S, Huang S and Sun YL: Epithelial-mesenchymal transition in pancreatic cancer: A review. Biomed Res Int 2017: 2646148, 2017.

36. Zheng X, Carstens JL, Kim J, Scheible M, Kaye J, Sugimoto H, Wu CC, LeBleu VS and Kalluri R: Epithelial-to-mesenchymal transition is dispensable for metastasis but induces chemoresistance in pancreatic cancer. Nature 527: 525-530, 2015.

37. Safa AR: Epithelial-mesenchymal transition: A hallmark in pancreatic cancer stem cell migration, metastasis formation, and drug resistance. J Cancer Metastasis Treat 6: 36, 2020.

38. Miura F, Takada T, Amano H, Yoshida M, Furui S and Takeshita K: Diagnosis of pancreatic cancer. HPB (Oxford) 8: 337-342, 2006.

39. Song SW, Cheng JF, Liu N and Zhao TH: Diagnosis and treatment of pancreatic metastases in 22 patients: A retrospective study. World J Surg Oncol 25: 299, 2014.

40. Nalankilli K, Kannuthurai S and Moss A: A modern approach to ERCP: Maintaining efficacy while optimising safety. Dig Endosc 28 (Suppl 1): S70-S76, 2016.

41. Tryliskyy Y and Bryce GJ: Post-ERCP pancreatitis: Pathophysiology, early identification and risk stratification. Adv Clin Exp Med 27: 149-154, 2018.

42. Dietrich CF and Christian J: Modern ultrasound imaging of pancreatic tumors. Ultrasonography 39: 105-113, 2020.

43. Gañán IM, Ríos LF, de Toledo LS, Mombila ME and Mendoza LH: Estadificación mediante tomografía computarizada del carcinoma de páncreas. Radiologia 60: 10-23, 2018 (In English, Spanish).

44. Azzaz HE, Abdullah MS and Habib RM: Role of multidetector computed tomography in evaluation of resectability of pancreatic cancer. Egyptian J Radiol Nuclear Med 52: 140, 2021.

45. Deng L, Ke X, He Z, Yang D, Gong H, Zhang Y, Jing X, Yao J and Chen J: A MSLN-targeted multifunctional nanoimmunoliposome for MRI and targeting therapy in pancreatic cancer. Int J Nanomedicine 7: 5053-5065, 2012.

46. Le K, Wang J, Zhang T, Guo Y, Chang H, Wang S and Zhu B: Overexpression of mesothelin in pancreatic ductal adenocarcinoma (PDAC). Int J Med Sci 17: 422-427, 2020.

47. Jha P and Bijan B: PET/CT for pancreatic malignancy: Potential and pitfalls. J Nucl Med Technol 43: 92-97, 2015.

48. Bhutani MS, Koduru P, Joshi V, Saxena P, Suzuki R, Irisawa A and Yamao K: The role of endoscopic ultrasound in pancreatic cancer screening. Endosc Ultrasound 5: 8-16, 2016.

49. Guijarro LG, Sanmartin-Salinas P, Pérez-Cuevas E, Toledo-Lobo MV, Monserrat J, Zoullas S, Sáez MA, Álvarez-Mon MA, Bujan J, Noguerales-Fraguas F, et al: Possible role of IRS-4 in the origin of multifocal hepatocellular carcinoma. Cancers (Basel) 23: 2560, 2021.

50. Majumder S, Chari ST and Ahlquist DA: Molecular detection of pancreatic neoplasia: Current status and future promise. World J Gastroenterol 28: 11387-11395, 2015. 
51. Kato K, Kamada H,Fujimori T, Aritomo Y, Ono M and Masaki T: Molecular biologic approach to the diagnosis of pancreatic carcinoma using specimens obtained by EUS-guided fine needle aspiration. Gastroenterol Res Pract 2012: 243524, 2012.

52. Hanada K, Minami T, Shimizu A, Fukuhara M, Yano S, Sasaki K, Koda M, Sugiyama K, Yonehara S and Yanagisawa A: Roles of ERCP in the early diagnosis of pancreatic cancer. Diagnostics (Basel) 7: 30, 2019.

53. Bispo M, Marques S, Rio-Tinto R, Fidalgo $\mathrm{P}$ and Devière J: The role of endoscopic ultrasound in pancreatic cancer staging in the Era of neoadjuvant therapy and personalised medicine. GE Port J Gastroenterol 28: 111-120, 2021.

54. Sun B and Hu B: The role of intraductal ultrasonography in pancreatobiliary diseases. Endosc Ultrasound 5: 291-299, 2016.

55. Puri R, Manrai M, Thandassery RB and Alfadda AA: Endoscopic ultrasound in the diagnosis and management of carcinoma pancreas. World J Gastrointest Endosc 25: 67-76, 2016.

56. Yamashita Y, Kato J, Ueda K, Nakamura Y, Kawaji Y, Abe H, Nuta J, Tamura T, Itonaga M, Yoshida T, et al: Contrast-enhanced endoscopic ultrasonography for pancreatic tumors. Biomed Res Int 2015: 491782, 2015

57. Pentyala S, Whyard T, Pentyala S, Muller J, Pfail J, Parmar S, Helguero CG and Khan S: Prostate cancer markers: An update. Biomed Rep 4: 263-268, 2016.

58. Gonssaud B, Goussot V, Berriolo-Riedinger A, Saïtta-Aribau E, Coutant C, Cochet A, Fumoleau P and Riedinger JM: Clinical value of CA 15-3 for detection of distant metastases in newly diagnosed breast cancer. Ann Biol Clin (Paris) 1: 421-429, 2017.

59. Bottoni P and Scatena R: The role of CA 125 as tumor marker: Biochemical and clinical aspects. Adv Exp Med Biol 867: 229-244, 2015

60. Coelho R, Silva M, Rodrigues-Pinto E, Cardoso H, Lopes S, Pereira P, Vilas-Boas F, Santos-Antunes J, Costa-Maia J and Macedo G: CA 19-9 as a marker of survival and a predictor of metastization in cholangiocarcinoma. GE Port J Gastroenterol 24: 114-121, 2017.

61. Poruk KE, Gay DZ, Brown K, Mulvihill JD, Boucher KM, Scaife CL, Firpo MA and Mulvihill SJ: The clinical utility of CA 19-9 in pancreatic adenocarcinoma: Diagnostic and prognostic updates. Curr Mol Med 13: 340-351, 2013.

62. Liu C, Deng S, Jin K, Gong Y, Cheng H, Fan Z, Qian Y, Huang Q, $\mathrm{Ni}$, Luo G and $\mathrm{Yu} \mathrm{X}$ : Lewis antigen-negative pancreatic cancer: An aggressive subgroup. Int J Oncol 56: 900-908, 2020

63. Salleh S, Thyagarajan A and Sahu RP: Exploiting the relevance of CA 19-9 in pancreatic cancer. J Cancer Metastasis Treat 6 : $31,2020$.

64. Amburn TL, Ginn M, Patel R, Pandalai PK, Kim J and Cavnar MJ: Clinical utility of symptoms and serum carbohydrate antigen (CA) 19-9 for detection of recurrence after curative intent resection of periampullary arcinoma. JACS 231: E122-E1123, 2020.

65. Pereira SP, Oldfield L, Ney A, Hart PA, Keane MG, Pandol SJ, Li D, Greenhalf W, Jeon CY, Koay EJ, et al: Early detection of pancreatic cancer. Lancet Gastroenterol Hepatol 5: 698-710, 2020.

66. Agrawal S: Potential prognostic biomarkers in pancreatic juice of resectable pancreatic ductal adenocarcinoma. World J Clin Oncol 10: 255-260, 2017.

67. Yip-Schneider MT, Soufi M, Carr RA, Flick KF, Wu H, Colgate CL and Schmidt CM: Performance of candidate urinary biomarkers for pancreatic cancer-correlation with pancreatic cyst malignant progression? Am J Surg 219: 492-495, 2020.

68. Lim JH, Park JS and Yoon DS: Preoperative fecal elastase-1 is a useful prognostic marker following curative resection of pancreatic cancer. HPB (Oxford) 19: 388-395, 2017.

69. Xu R, Xu Q, Huang G, Yin X, Zhu J,Peng Y and Song J: Combined analysis of the aberrant epigenetic alteration of pancreatic ductal adenocarcinoma. Biomed Res Int 28: 9379864, 2019.

70. Rückert F, Pilarsky C and Grützmann R: Serum tumor markers in pancreatic cancer-recent discoveries. Cancers (Basel) 2: $1107-1124,2010$

71. Zhang J, Wang Y, Zhao T, Li Y, Tian L, Zhao J and Zhang J: Evaluation of serum MUC5AC in combination with CA19-9 for the diagnosis of pancreatic cancer. World J Surg Oncol 7: 31 , 2020.

72. Kim H, Kang KN, Shin YS, Byun Y, Han Y, Kwon W, Kim CW and Jang JY: Biomarker panel for the diagnosis of pancreatic ductal adenocarcinoma. Cancers (Basel) 1: 1443, 2020.

73. Loosen SH, Neumann UP, Trautwein C, Roderburg C and Luedde T: Current and future biomarkers for pancreatic adenocarcinoma. Tumor Biol 39: 101042831769223, 2017.
74. Lee HS, Jang CY, Kim SA, Park SB, Jung DE, Kim BO, Kim HY, Chung MJ, Park JY and Bang S, et al: Combined use of CEMIP and CA 19-9 enhances diagnostic accuracy for pancreatic cancer. Sci Rep 21: 3383, 2018.

75. Kim J, Bamlet WR, Oberg AL, Chaffee KG, Donahue G, Cao XJ, Chari S, Garcia BA, Petersen GM and Zaret KS: Detection of early pancreatic ductal adenocarcinoma with thrombospondin-2 and CA19-9 blood markers. Sci Transl Med 12: eaah5583, 2017.

76. Jenkinson C, Elliott VL, Evans A, Oldfield L, Jenkins RE, O'Brien DP, Apostolidou S, Gentry-Maharaj A, Fourkala EO, Jacobs IJ, et al: Decreased serum thrombospondin-1 levels in pancreatic cancer patients up to 24 months prior to clinical diagnosis: Association with diabetes mellitus. Clin Cancer Res 22: 1734-1743, 2016.

77. Radon TP, Massat NJ, Jones R, Alrawashdeh W, Dumartin L, Ennis D, Duffy SW, Kocher HM, Pereira SP, posthumous LG, et al: Identification of a three-biomarker panel in urine for early detection of pancreatic adenocarcinoma. Clin Cancer Res 1: 3512-3521, 2015.

78. Benassai G, Quarto G, Perrotta S, Furino E, Benassai GL, Amato B, Bianco T, Palma GD and Forestieri P: Long-term survival after curative resection for pancreatic ductal adenocarcinoma-surgical treatment. Int J Surg 21 (Suppl 1): S1-S3, 2015

79. Jeekel H: Prognostic factors following curative resection for pancreatic adenocarcinoma. Ann Surg 240: 384, 2004.

80. Brunner M, Wu Z, Krautz C, Pilarsky C, Grützmann R and Weber GF: Current clinical strategies of pancreatic cancer treatment and open molecular questions. Int J Mol Sci 13: 4543, 2019.

81. Ho CK, Kleeff J, Friess H and Büchler MW: Complications of pancreatic surgery. HPB (Oxford) 7: 99-108, 2005.

82. Andren-Sandberg A: Complications of pancreatic surgery. N Am J Med Sci 3: 531-535, 2011.

83. Liu M, Ji S, Xu W, Liu W, Qin Y, Hu Q, Sun Q, Zhang Z, Yu X and $\mathrm{Xu} \mathrm{X}$ : Laparoscopic pancreaticoduodenectomy: Are the best times coming? World J Surg Oncol 10: 81, 2019.

84. Zhu R, Cao Z, Qiu J and Zhang T: Minimally invasive pancreatic surgery: An upward spiral. Laparoscopic, Endoscopic and Robotic Surgery 3: 29-33, 2020.

85. Magge D, Zureikat A, Hogg M and Zeh HJ III: Minimally invasive approaches to pancreatic surgery. Surg Oncol Clin N Am 25: 273-286, 2016

86. Liu R, Wakabayashi G, Palanivelu C, Tsung A, Yang K, Goh BK, Chong CC, Kang CM, Peng C, Kakiashvili E, et al: International consensus statement on robotic pancreatic surgery. Hepatobiliary Surg Nutr 8: 345-360, 2019.

87. Rashid OM, Mullinax JE, Pimiento JM, Meredith KL and Malafa MP: Robotic whipple procedure for pancreatic cancer: The moffitt cancer center pathway. Cancer Control 22: 340-351, 2015.

88. Rehders A, Stoecklein NH, Güray A, Riediger R, Alexander A and Knoefel WT: Vascular invasion in pancreatic cancer: Tumor biology or tumor topography? Surgery 152 (Suppl 1): S143-S151, 2012.

89. Zaky AM, Wolfgang CL, Weiss MJ, Javed AA, Fishman EK and Zaheer A: Tumor-vessel relationships in pancreatic ductal adenocarcinoma at multidetector CT: Different classification systems and their influence on treatment planning. Radiographics 37: 93-112, 2017.

90. Dua MM, Tran TB, Klausner J, Hwa KJ, Poultsides GA, Norton JA and Visser BC: Pancreatectomy with vein reconstruction: Technique matters. HPB (Oxford) 17: 824-831, 2015.

91. Delpero JR and Sauvanet A: Vascular resection for pancreatic cancer: 2019 French recommendations based on a literature review from 2008 to 6-2019. Front Oncol 4: 40, 2020.

92. Sgroi MD, Narayan RR, Lane JS, Demirjian A, Kabutey NK, Fujitani RM and Imagawa DK: Vascular reconstruction plays an important role in the treatment of pancreatic adenocarcinoma. J Vasc Surg 61: 475-480, 2015

93. Rebelo A, Michalski C, Ukkat J and Kleeff J: Pancreatic cancer surgery with vascular resection: Current concepts and perspectives. J Pancreatol 2: 1-5, 2019.

94. Perinel $\mathrm{J}$ and Adham M: Palliative therapy in pancreatic cancer-palliative surgery. Transl Gastroenterol Hepatol 7: 28, 2019.

95. Pencovich N, Orbach L, Lessing Y, Elazar A, Barnes S, Berman P, Blachar A, Nachmany I and Sagie B: Palliative bypass surgery for patients with advanced pancreatic adenocarcinoma: Experience from a tertiary center. World J Surg Oncol 1: 63, 2020. 
96. Assfalg V, Hüser N, Michalski C, Gillen S, Kleeff J and Friess H: Palliative interventional and surgical therapy for unresectable pancreatic cancer. Cancers (Basel) 14: 652-661, 2011.

97. Pezzilli R: Neuropathic pain in pancreatic cancer: An update of the last five years. Gastroenterol Insights 12: 302-309, 2021.

98. Zhong W, Yu Z, Zeng JX, Lin Y, Yu T, Min XH, Yuan YH and Chen QK: Celiac plexus block for treatment of pain associated with pancreatic cancer: A meta-analysis. Pain Pract 14: 43-51, 2014

99. Springfeld C, Jäger D, Büchler MW, Strobel O, Hackert T, Palmer DH and Neoptolemos JP: Chemotherapy for pancreatic cancer. Presse Med 48: e159-e174, 2019.

100. Hajatdoost L, Sedaghat K, Walker EJ, Thomas J and Kosari S: Chemotherapy in pancreatic cancer: A systematic review. Medicina (Kaunas) 11: 48, 2018.

101. Marco MD, Cicilia RD, Macchini M, Nobili E, Vecchiarelli S, Brandi G and Biasco G: Metastatic pancreatic cancer: Is gemcitabine still the best standard treatment? (Review). Oncol Rep 23: 1183-1192, 2010

102. Martín AM, Hidalgo M, Alvarez R, Arrazubi V, Martínez-Galán J, Salgado M, Macarulla T and Carrato A: From first line to sequential treatment in the management of metastatic pancreatic cancer. J Cancer 30: 1978-1988, 2018.

103. Conroy T, Hammel P, Hebbar M, Abdelghani MB, Wei AC Raoul JL, Choné L, Francois E, Artru P, Biagi JJ, et al: FOLFIRINOX or gemcitabine as adjuvant therapy for pancreatic cancer. N Engl J Med 20: 2395-2406, 2018.

104. Azam F, Latif MF, Faroog A. Tirmazy SH, AlShahrani S, Bashir S and Bukhari N: Performance status assessment by using ECOG (Eastern Cooperative Oncology Group) score for cancer patients by oncology healthcare professionals. Case Rep Oncol 25: 728-736, 2019

105. Lee J, Lee JC, Gromski MA, Kim HW, Kim J, Kim J and Hwang JH: Clinical outcomes of FOLFIRINOX in locally advanced pancreatic cancer: A single center experience. Medicine (Baltimore) 97: e13592, 2018.

106. Yoo C, Kang J, Kim KP, Lee JL, Ryoo BY, Chang HM, Lee SS, Park DH, Song TJ, Seo DW, et al: Efficacy and safety of neoadjuvant FOLFIRINOX for borderline resectable pancreatic adenocarcinoma: Improved efficacy compared with gemcitabine-based regimen. Oncotarget 11: 46337-46347, 2017.

107. Tahara J, Shimizu K, Otsuka N, Akao J, Takayama Y and Tokushige K: Gemcitabine plus nab-paclitaxel vs. FOLFIRINOX for patients with advanced pancreatic cancer. Cancer Chemother Pharmacol 82: 245-250, 2018

108. Ma P and Mumper RJ: Paclitaxel nano-delivery systems: A comprehensive review. J Nanomed Nanotechnol 18: 1000164 2013

109. Chatterjee M, Ben-Josef E, Robb R, Vedaie M, Seum S Thirumoorthy K, Palanichamy K, Harbrecht M, Chakravarti A and Williams TM: Caveolae-mediated endocytosis is critical for albumin cellular uptake and response to albumin-bound chemotherapy. Cancer Res 1: 5925-5937, 2017.

110. Yardley DA: Nab-paclitaxel mechanisms of action and delivery. J Control Release 28: 365-372, 2013.

111. Kang J, Hwang I, Yoo C, Kim KP, Jeong JH, Chang HM, Lee SS, Park DH, Song TJ, Seo DW, et al: Nab-paclitaxel plus gemcitabine versus FOLFIRINOX as the first-line chemotherapy for patients with metastatic pancreatic cancer: Retrospective analysis. Invest New Drugs 36: 732-741, 2018.

112. Kim G: Nab-paclitaxel for the treatment of pancreatic cancer Cancer Manag Res 16: 85-96, 2017.

113. Wolfe AR, Prabhakar D, Yildiz VO, Cloyd JM, Dillhoff M, Abushahin L, Diaz DA, Miller ED, Chen W, Frankel WL, et al: Neoadjuvant-modified FOLFIRINOX vs. nab-paclitaxel plus gemcitabine for borderline resectable or locally advanced pancreatic cancer patients who achieved surgical resection. Cancer Med 9: 4711-4723, 2020.

114. Sarabi M, Mais L, Oussaid N, Desseigne F, Guibert P and Fouchardiere CD: Use of gemcitabine as a second-line treatment following chemotherapy with folfirinox for metastatic pancreatic adenocarcinoma. Oncol Lett 13: 4917-4924, 2017.

115. Kordes M, Yu J, Malgerud O, Liljefors MG and Löhr JM: Survival benefits of chemotherapy for patients with advanced pancreatic cancer in a clinical real-world cohort. Cancers (Basel) 7: 1326, 2019.

116. Oba A, Ho F, Bao QR, Al-Musawi MH, Schulick RD and Chiaro MD: Neoadjuvant treatment in pancreatic cancer. Front Oncol 28: 245, 2020
117. Versteijne E, Vogel JA, Besselink MG, Busch OR, Wilmink JW, Daams JG, van Eijck CHJ, Koerkamp BG, Rasch CR, van Tienhoven $\mathrm{G}$, et al: Meta-analysis comparing upfront surgery with neoadjuvant treatment in patients with resectable or borderline resectable pancreatic cancer. Br J Surg 105: 946-958, 2018

118. Seufferlein T and Ettrich TJ: Treatment of pancreatic cancer-neoadjuvant treatment in resectable pancreatic cancer (PDAC). Transl Gastroenterol Hepatol 27: 21, 2019.

119. Müller PC, Frey MC, Ruzza CM, Nickel F, Jost C, Gwerder C, Hackert T, Z'graggen $\mathrm{K}$ and Kessler U: Neoadjuvant chemotherapy in pancreatic cancer: An appraisal of the current high-level evidence. Pharmacology 106: 143-153, 2021.

120. Hall WA and Karyn A: Goodman: Radiation therapy for pancreatic adenocarcinoma, a treatment option that must be considered in the management of a devastating malignancy. Radiat Oncol 26: 114, 2019.

121. Tchelebi LT, Zaorsky NG, Rosenberg JC, Sharma NK, Tuanquin LC, Mackley HB and Ellis RJ: Reducing the toxicity of radiotherapy for pancreatic cancer with magnetic resonance-guided radiotherapy. Toxicol Sci 1: 19-23, 2020

122. Buwenge M, Macchia G, Arcelli A, Frakulli R, Fuccio L, Guerri S, Grassi E, Cammelli S, Cellini F and Morganti AG: Stereotactic radiotherapy of pancreatic cancer: A systematic review on pain relief. J Pain Res 4: 2169-2178, 2018

123. Palta M, Godfrey D, Goodman KA, Hoffe S, Dawson LA, Dessert D, Hall WA, Herman JM, Khorana AA, Merchant N, et al: Radiation therapy for pancreatic cancer: Executive summary of an ASTRO clinical practice guideline. Pract Radiat Oncol 9: 322-332, 2019.

124. Shah AP, Strauss JB and Abrams RA: Review and commentary on the role of radiation therapy in the adjuvant management of pancreatic cancer. Am J Clin Oncol 33: 101-106, 2010.

125. Jiang W, Haque W, Verma V, Butler EB and Teh BS: Neoadjuvant stereotactic body radiation therapy for nonmetastatic pancreatic adenocarcinoma. Acta Oncol 58: 1259-1266, 2019.

126. Glicksman RM, Chung H, Myrehaug S, Erler D, Korol R, Karotki A, Taggar A and Ung YC: Stereotactic radiotherapy for pancreatic cancer: A single-institution experience. Cureus 23: $\mathrm{e} 10618,2020$

127. Gou Q, Dong C, Xu H, Khan B, Jin J, Liu Q, Shi J and Hou Y: PD-L1 degradation pathway and immunotherapy for cancer. Cell Death Dis 6: 955, 2020

128. Jiang T, Shi T, Zhang H, Hu J, Song Y, Wei J, Ren S and Zhou C: Tumor neoantigens: From basic research to clinical applications. J Hematol Oncol 6: 93, 2019.

129. Das M, Zhou X, Liu Y, Das A, Vincent BG, Li J, Liu R and Huang L: Tumor neoantigen heterogeneity impacts bystander immune inhibition of pancreatic cancer growth. Transl Oncol 13: 100856, 2020.

130. Chi J, Patel R, Rehman H, Goyal S and Saif MW: Recent advances in immunotherapy for pancreatic cancer. J Cancer Metastasis Treat 6: 43, 2020

131. Chen H, Yang G, Xiao J, Zheng L, You L and Zhang T: Neoantigen-based immunotherapy in pancreatic ductal adenocarcinoma (PDAC). Cancer Lett 10: 12-19, 2020.

132. Looi CK, Chung FFL, Leong CO, Wong SF, Rosli R and Mai CW: Therapeutic challenges and current immunomodulatory strategies in targeting the immunosuppressive pancreatic tumor microenvironment. J Exp Clin Cancer Res 15: 162, 2019.

133. Royal RE, Levy C, Turner K, Mathur A,Hughes M, Kammula US, Sherry RM, Topalian SL, Yang JC, Lowy I and Rosenberg SA: Phase 2 trial of single agent ipilimumab (Anti-CTLA-4) for locally advanced or metastatic pancreatic adenocarcinoma. J Immunother 33: 828-833, 2010.

134. Taube JM, Klein A, Brahmer JR, Xu H, Pan X, Kim JH, Chen L, Pardoll DM, Topalian SL and Anders RA: Association of PD-1, PD-1 ligands, and other features of the tumor immune microenvironment with response to anti-PD-1 therapy. Clin Cancer Res 1: 5064-5074, 2014

135. Amin S, Baine M, Meza J, Alam M and Lin C: The impact of immunotherapy on the survival of pancreatic adenocarcinoma patients who do not receive definitive surgery of the tumor. Clin Transl Radiat Oncol 7: 34-40, 2020.

136. Brouwer TP, Vahrmeijer AL and de Miranda NF: Immunotherapy for pancreatic cancer: Chasing the light at the end of the tunnel. Cell Oncol (Dordr) 44: 261-278, 2021

137. Krishnamoorthy M, Lenehan JG, Burton JP and Vareki SM: Immunomodulation in pancreatic cancer. Cancers (Basel) 12: 3340, 2020. 
138. Sideras K, Braat H, Kwekkeboom J, van Eijck $\mathrm{CH}$ Peppelenbosch MP, Sleijfer S and Bruno M: Role of the immune system in pancreatic cancer progression and immune modulating treatment strategies. Cancer Treat Rev 40: 513-522, 2014.

139. Amedei A, Niccolai E and Prisco D: Pancreatic cancer: Role of the immune system in cancer progression and vaccine-based immunotherapy. Hum Vaccin Immunother 10: 3354-3368, 2014.

140. Luo W, Yang G, Luo W, Cao Z, Liu Y, Qiu J, Chen G, You L, Zhao F, Zheng L and Zhang T: Novel therapeutic strategies and perspectives for metastatic pancreatic cancer: Vaccine therapy is more than just a theory. Cancer Cell Int 4: 66, 2020.

141. Akce M, Zaidi MY, Waller EK, El-Rayes BF and Lesinski GB: The potential of CAR T cell therapy in pancreatic cancer. Front Immunol 25: 2166, 2018.

142. Penack O and Koenecke C: Complications after CD19+ CAR T-cell therapy. Cancers (Basel) 19: 3445, 2020.

143. Golan T, Hammel P, Reni M, Cutsem EV, Macarulla T, Hall MJ, Park JO, Hochhauser D, Arnold D, Oh DY, et al: Maintenance olaparib for germline brca-mutated metastatic pancreatic cancer. N Engl J Med 25: 317-327, 2019.
144. Picozzi V, Alseidi A, Winter J, Pishvaian M, Mody K Glaspy J, Larson T, Matrana M, Carney M, Porter S, et al: Gemcitabine/nab-paclitaxel with pamrevlumab: A novel drug combination and trial design for the treatment of locally advanced pancreatic cancer. ESMO Open 5: e000668, 2020.

145. Alistar A, Morris BB, Desnoyer R, Klepin HD, Hosseinzadeh K, Clark C, Cameron A, Leyendecker J, D'Agostino R Jr, Topaloglu U, et al: Safety and tolerability of the first-in-class agent CPI-613 in combination with modified FOLFIRINOX in patients with metastatic pancreatic cancer: A single-centre, open-label, dose-escalation, phase 1 trial. Lancet Oncol 18: $770-778,2017$

c) (ㅇ) $\ominus$ This work is licensed under a Creative Commons EY NC ND Attribution-NonCommercial-NoDerivatives 4.0 International (CC BY-NC-ND 4.0) License. 